\title{
GENERATING INSIGHT IN DISCRETE EVENT SIMULATION AND AGENT-BASED MODELLING: EXPERIMENTAL EVIDENCE
}

\author{
Hilya Mudrika Arini \\ Universitas Gadjah Mada \\ Yogyakarta, Indonesia \\ hilya.mudrika@ugm.ac.id
}

Nur Aini Masruroh

Universitas Gadjah Mada

Yogyakarta, Indonesia

aini@ugm.ac.id

\author{
Nurul Lathifah
}

Universitas Gadjah Mada

Yogyakarta, Indonesia

nunurullathifah@gmail.com

\section{Laela Dewi Maharani}

Universitas Gadjah Mada

Yogyakarta, Indonesia

laela.dmaharani@gmail.com

\begin{abstract}
This study aims to understand the difference between discrete-event simulation (DES) and agent-based modelling (ABM) user and non-user in generating insight. In total, 41 undergraduate students who were DES and ABM users and non-users participated in the experiment and given task of solving problem using simulation models. The insight generation is measured by four variables: (1) task performance, (2) problem understanding, (3) discontinuity in thinking, and (4) change in problem understanding. This study concludes that there are no significant differences between DES and ABM users and non-users in generating insight particularly for simulation with low complexity as utilised in this study. However, both ABM users and non-users had higher solution rates, indicating higher insight occurrence, than DES users and non-users. Since this study limited to simulation with low complexity, there is a room of improvement for further study to investigate different complexity level of the system simulated in generating insight.
\end{abstract}

Keywords: Behavioural Operational Research, Generating Insight, Discrete Event Simulation, AgentBased Modelling

\section{INTRODUCTION}

Simulation has been used in many service and manufacturing sector projects. It is considered a costeffective method for improving, investigating, and evaluating the performance of resource allocation and alternative operating policies (Chung, 2004). In simulation, to test and enhance system performance, a complex system is simplified using a computer program. This condition helps by creating opportunities to test various concepts and ideas, which have been called scenarios. Due to the advantages it offers, many simulation techniques have been developed.

One of the simulation techniques that is widely used is discrete event simulation (DES). DES is a simulation technique that can help in decision-making and problem-solving. In DES, the real world is represented by simulating its dynamics on an event-by-event basis (Babulak \& Wang, 2010). However, DES helps in modelling the system by focusing on its events or processes only. In addition to DES, agent-based modeling $(\mathrm{ABM})$ is another simulation technique that is popular for modelling people's behaviour. ABM consists of agents that can interact with and influence each other to create an emergence of behaviour. In addition, these agents can learn from their experiences and adjust their actions to better suit their environment (Macal \& North, 2010). According to Pegden et al. (1995), the 
purpose of ABM is to gain insight into the operation of the system. Aside from Pegden et al. (1995), Leemis and Park (2004) have also stated that the purpose of DES is insight, which means getting a better understanding of how the system operates and responds to changes.

Both DES and ABM appear to be simulation approaches that could model and simulate human behaviour (Majid et al., 2016). As Dubiel and Tsimhoni (2005) identify, there is some research that integrates DES and ABM. Some of this previous research has sought to compare the implementation of DES and ABM in different fields, including operations research and implementation of new policies (Zankoul et al., 2015). Thus, it does not rule out the possibility that DES and ABM can be compared in terms of insight generation.

Insight is defined as novel ideas that provide a better understanding of a phenomenon. Gogi et al. (2016) explained that, in simulation, insight occurs when people who have used a simulation model discover how to improve a system's performance by testing a 'what-if' scenario. In previous research by Gogi et al. (2016), the analysis of insight occurrence in DES has been performed. According to Gogi et al. (2016), simulation can help in generating insight even though statistically there is no significant difference between the use of animated displays and the statistical results from simulation model. This study also explained that the result obtained depends on the case simulated and the type of simulation, where different simulation cases and different simulation types might produce different results.

Accordingly, this study attempts to develop the research by Gogi et al. (2016) by utilising different cases and comparing the insight occurrence in DES and in ABM. Both DES and ABM can provide an animated display that is easier to understand, as well as the statistical results of the model that has been run to aid comprehension and assist in further data processing. As both DES and ABM are intended to provide insight, in this paper, empirical evidence of the difference in insight generation through simulation, in particular using DES and ABM, is provided by comparing the users and non-users of each simulation technique.

\section{LITERATURE REVIEW}

Simulation is usually performed using a computer program or simulation software that models the behaviour of real systems, and is used to analyse the system's behaviour and then formulate a policy decision (Chung, 2004). Most of the recent research on simulation discusses the implementation, development, and improvement of simulation. Some scholars have also conducted research to improve the verification and validation process of simulation, to ensure the accuracy of the modelling.

Bannet et al. (2013) explains that the usefulness of a model is not only about the accuracy of the model, but it can also be evaluated by the user. However, studies evaluating simulation models are limited, particularly studies that take into account the insight generation in simulation (Gogi et al., 2016). However, Aalst and Voorhoeve (2000) state that, through creating a simulation model, the insight generated from simulating existing or proposed future situations can be useful.

Gogi et al. (2016) explain that insight occurrence can be measured based on four variables: (1) task performance, (2) problem understanding, (3) discontinuity in thinking, and (4) change in problem understanding. Task performance is defined as the ability of the problem-solver to achieve the goals of the task, whilst problem understanding can be understood as the ability of the problem-solver to solve the problem by using a simulation model. Discontinuity in thinking occurs when the problem-solver follows a procedure that leads to multiple possible solutions for the problem. A change in understanding is defined as the ability of the problem-solver to gain a better understanding, and can be measured by comparing the understanding before and after using the simulation model.

According to Gogi et al. (2016), simulation plays an important role in the process of generating insight. Thus, this study will expand on Gogi et al.'s (2016) research to provide empirical evidence related to insight generation in simulation, especially DES and ABM. In this study, the behaviours of users and non-users of simulation will be analysed. Two different cases will be used order to represent DES and ABM.

\section{METHODOLOGY}

This section will provide more detail about the experiment will be described. This includes the design of experiment, details about participants, the materials used for the experiment, procedures carried out, as well as the details of and results from the pilot study. 


\subsection{Experiment Design}

In this study, two simulations were used, DES and ABM. Each experiment of simulation model consisted of users and non-users of the simulation who were involved separately. The experiments were divided into three sections: the pre-test, treatment, and post-test.

In order to avoid discrepancies in the information given, the data collection process was carried out using a pre-prepared script. For the treatment section, an initial simulation model was provided and participants were required to reuse the model and develop the scenario until the optimal solution had been identified and the goal that had been defined by the researcher was achieved. The use of simulation model used only depends on the model given and had nothing to do with the real conditions of the system. Thus, the only factors considered were experience of using simulations not the knowledge of real systems. Finally, the analysis was carried out by comparing the results of the pre-test and post-test as well as the results from each scenario that was built by the participants.

\subsection{Participants}

This study included a total of 41 undergraduate students in Indonesia. The participants consisted of 11 DES users, 10 DES non-users, 10 ABM users, and 10 ABM non-users. DES users were students who had studied DES, whilst DES non-users were students who had not studied DES. Similarly, the ABM users were students who had studied ABM, whilst ABM non-users were students who had not studied ABM. The participants were tasked with solving a problem using simulation, either with DES or ABM.

In order to ensure that the participants' performances could be compared, this experiment used homogeneous participants, where the participants of this experiment had an equal standard of their knowledge in simulation. To achieve this, certain requirements needed to be met to become a participant. For user participants, these were: the participant must be an undergraduate student that have a "good" academic score, demonstrated by a minimum score of B for courses to the case study (operational research, system modelling, and simulation) and a minimum GPA of 3.00 out of 4.00. Meanwhile, for non-user participant, the different requirement were located on the absent on taking system modelling and simulation course.

\subsection{Materials}

This section will describe the materials used during the experiment. This includes the experimental protocol, an explanation of the case study used, and an explanation of the simulation model used in the experiment.

\subsubsection{Experiment Protocol}

The protocol used in this study is an adaptation of the experiment protocol used previously by Gogi (2016). The protocol set out the steps that had to be followed by participants according to the condition they were assigned to, whether DES or ABM. In the protocol, general instructions were given, as well as an explanation of the case study and instructions for using the simulation software. In addition, it contained a pre-test questionnaire and a post-test questionnaire.

\subsubsection{Case Study}

Two case studies were used in this experiment. For the DES condition, the case study used was about the use of a student corner (SC) facility in the Mechanical and Industrial Engineering Department of Universitas Gadjah Mada (UGM). The SC is one of the facilities provided by UGM to support the students in having discussions and carrying out other learning activities on campus. Currently, there are eight round tables with capacity of five people per table and three rectangular tables with capacity of twelve people per table. The total number of students in the Mechanical and Industrial Engineering Department at UGM is approximately 280 students. The process flow of this case study is that, when a student who wants to use the SC enters the campus, they will go to the SC area where they will see the SC condition, whether there is an empty chair or not. When there is a chair available, the student will sit and use the SC. However, when all the seats in the SC are full, it is assumed that the student will go somewhere else, namely the reference room, which has 50 seats. The students will wait in the reference room and then, when there is a chair available, return to the SC. 
The DES model uses three entities: students, the SC area, and the reference room. The model has five main activities: the students come to the SC; students look for an empty chair in the SC on the floor; students look for an empty chair in the SC on the second floor; students use the SC; students use the reference room. The activity cycle diagram (ACD) for this model can be seen in Figure 1.

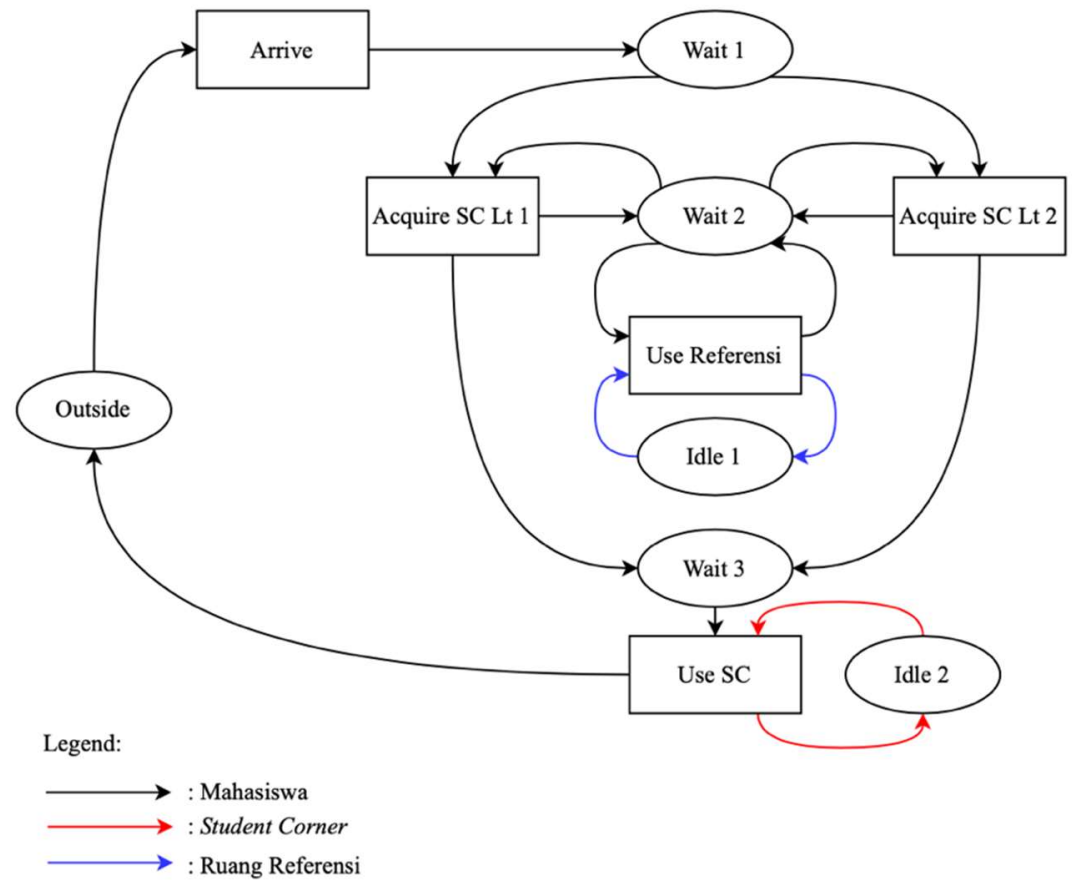

Figure 1 Activity Cycle Diagram for DES Model

For ABM, the case study used was about the HIV virus, and can be found in the Netlogo library. HIV can spread in various ways, such as by needle-sharing among injecting drug users, through blood transfusions, from HIV-infected women to their babies, and from sexual contact. The models examine the emergent effects of four aspects of sexual behaviour, namely: (1) average coupling tendency, (2) average commitment or amount of time that couple in the population stays together, (3) average condom use or the tendency of the population to use a condom; and (4) average test frequency or tendency to get tested for HIV. In this model, three colours are used: green, which represents uninfected individuals; blue, which represents infected individuals whose infection is unknown; and red, which represents infected individuals whose infection is known.

The ABM model consist of two entities: people, and the environment in which the people move. The state variables for the people are divided into: people who are uninfected, infected-unknown, and infected-known. At the initiation of the model, there are around 50-500 people who can be set in the model. In this model, people in the population will interact and form a 'couple'; those people who are not in couples will wander around the environment until they find a coupling. Considering the number of entities, state variables, purpose, and processes in the model, it can be said that the model complexity is simple.

From the descriptions above, it is clear that both the DES and ABM models used in this experiment are simple in terms of model complexity. The DES model consists of three entities with a simple flow and activity, whilst the ABM model consists of two entities with the purpose of examining the spread of a virus in a small isolated human population. Thus, due to having the same level of model complexity, the two simulation models can be compared.

\subsubsection{Simulation Model}

The SC simulation model was developed by Hersetiawan et al. (2019) using Flexsim software. The simulation model presents the process of SC daily use based on the number of uses for each table and the different usage times. This case focuses only on the use of SC facilities, whilst the use of the reference room, which also exists in the model, was ignored because it is considered a waiting area. The 
SC simulation model in Flexsim can be seen in Figure 2. In the model, the animated display and statistical results generated from the simulation run can be analysed by participants.

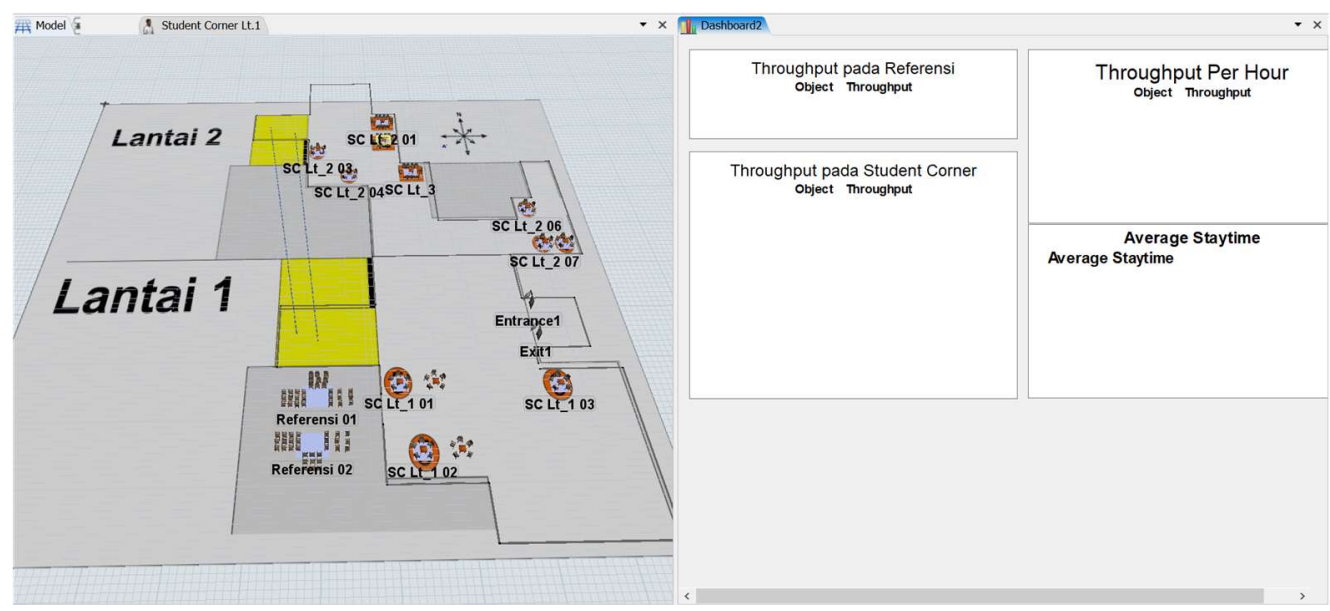

Figure 2 Interface of the Student Corner Model in Flexsim

The simulation model used for ABM was the HIV model that exists in the Netlogo software library, and can be seen in Figure 3. This HIV model was developed by Wilensky (1997). The model simulates the spread of the HIV virus through sexual transmission in a small, isolated human population. Thus, the model illustrates the effects of certain sexual practices across a population. In this model, several variables are used, which are: initial person, average coupling tendency, average commitment, average condom use, and average test frequency. The output is the rate of HIV infection in the population (as a percentage) over a certain period of time.

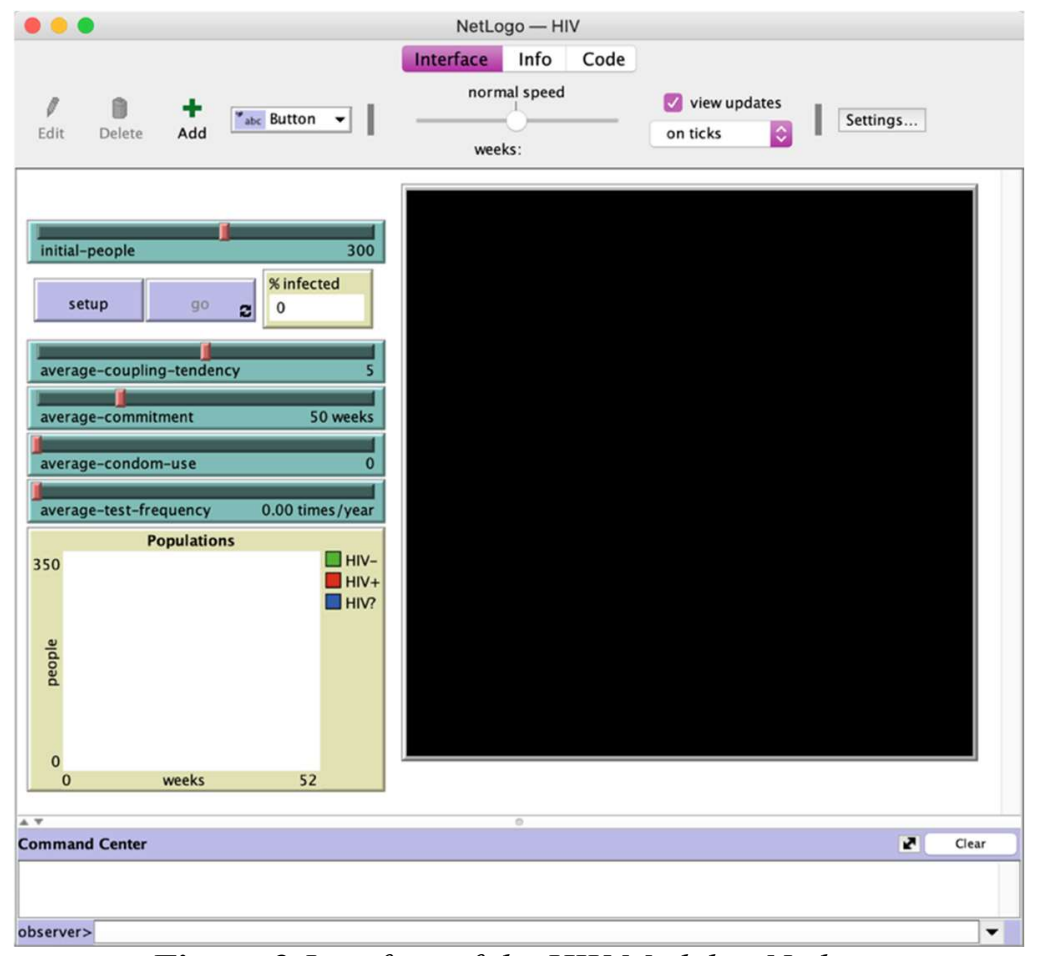

Figure 3 Interface of the HIV Model in Netlogo

\subsection{Experiment Procedure}

The experiments in both DES and ABM were conducted using the same procedure: they were conducted online using web and video conferencing tools. In the experiment, each participant had a private session with the authors. At the beginning of the session, the experiment to be carried out was explained to participants. Then, the participants were asked to read general instructions and complete the consent 
form. Next, the participant was asked to read the case study and complete a pre-test questionnaire that asked their opinion about the problem of the case study and its causes. This pre-test questionnaire was used to analyse the participants' problem understanding before using simulation.

Next, the participants were given a period of time to read the instructions for how to use the simulation software. Then, they were asked to run and work on the SC simulation that had been modelled in Flexsim or the HIV model in the Netlogo software. The participants were asked to analyse the model by creating scenarios in order to improve the system or solve the problem in the model. In Flexsim, the scenarios were created by changing the number of tables from the previous model. In Netlogo, the scenarios were created by changing the four parameters that were available in the model average coupling tendency, average commitment, average condom use, and average test frequency. This process was called the solving session and had to be completed in 30 minutes. After the participants had completed the solving session, they were asked to complete the post-test questionnaire, which contained some questions about the scenarios that could provide the best solutions for the problem. This post-test questionnaire was used to assess participants' understanding of the problem after using the simulation.

\section{RESULTS AND DISCUSSION}

In this study, four variables were analysed in order to assess the insight occurrence (Gogi et al., 2016). The variables are: (1) task performance, (2) problem understanding, (3) discontinuity in thinking, and (4) change in problem understanding. These variables were used to analyse the differences in insightgenerating processes among users and non-users of DES and ABM.

\subsection{Task Performance}

The task performance was measured based on whether or not the participant achieved the goal of the task, as determined by the participant's answers in the post-test questionnaire. Depending on their answers, the participants were classified into a 'solver' group and a 'non-solver' group. The solver group consisted of participants who were able to submit the optimal solution for the problem. The participants who did not submit the optimal solution, or submitted a solution that did not solve the problem, were classified into the non-solver group.

In DES, participants who submitted scenarios that involved increasing the capacity of the SC were considered solvers. The participants who reduced the capacity of the existing SC were categorised as non-solvers, because they had not overcome the existing problem. In ABM, participants who could achieve the goal set at the outset, which was to have a maximum of $2.84 \%$ of the population infected in week 156, were considered solvers. The difference in the number of solvers among users and nonusers of DES and ABM can be seen in Table 1 below.

Table 1 Proportion of Solvers Among Users and Non-users of DES and ABM

\begin{tabular}{l||cccc||cc}
\hline \multicolumn{1}{c||}{ Solvers } & \multicolumn{3}{c}{ DES } & \multicolumn{2}{c||}{ ABM } & \multicolumn{2}{c}{ Total } \\
& $\boldsymbol{n}$ & $\mathbf{\%}$ & $\boldsymbol{n}$ & $\mathbf{\%}$ & $\boldsymbol{n}$ & $\mathbf{\%}$ \\
\hline Users & 9 & $53 \%$ & 10 & $50 \%$ & 19 & $51 \%$ \\
Non-users & 8 & $47 \%$ & 10 & $50 \%$ & 18 & $49 \%$ \\
\hline \hline Total & 17 & $100 \%$ & 20 & $100 \%$ & 37 & $100 \%$ \\
\hline
\end{tabular}

In order to test the difference of task performance that the problem-solving process for users and non-users of DES and ABM, the chi-square test was adopted to compare the solution rate for both conditions, DES and ABM. The result of the statistical test showed a p-value of 0.8584 . From this result, with a significance level of 0.05 , it can be concluded that there is no significant difference between the problem-solving process for users and non-users of DES and ABM.

\subsection{Problem Understanding}

Participants' problem understanding was measured in order to determine whether the participants were solving the problem by generating insight or by intuition (Gogi et al., 2016). If the participants were 
able to justify their solution at the end of the session after running the simulation model, the participants were identified to have solved the problem by generating insight. This problem understanding was measured using two open-ended questions that asked about the reasons why the chosen scenario could solve the problem and what the user of the system should do in order to achieve the target. The participants' answers were grouped into three categories: inaccurate, incomplete, and complete.

The inaccurate category includes incorrect or unclear actions and inaccurate justifications of the reasons the proposed scenario can solve the problem. The incomplete category includes the answers of participants who did not directly solve the problem. The complete category includes the answers of participants who directly solved and fully recognised the cause of the problem and the action that must be taken to solve the problem.

Table 2 Problem Understanding After Running Simulation Model

\begin{tabular}{l||cccc||c}
\hline \multicolumn{1}{c||}{$\begin{array}{c}\text { Post-test Problem } \\
\text { Understanding }\end{array}$} & \multicolumn{2}{c|}{ DES $(\mathbf{n}=\mathbf{1 7})$} & \multicolumn{2}{c||}{ ABM $(\mathbf{n}=\mathbf{2 0})$} & \multirow{2}{*}{ Total } \\
\hline Inaccurate & 0 & 1 & 0 & 0 & 1 \\
Incomplete & 1 & 0 & 0 & 1 & 2 \\
Complete & 8 & 7 & 10 & 9 & 34 \\
\hline \hline Total & 9 & 8 & 10 & 10 & 37 \\
\hline
\end{tabular}

From Table 2, it can be seen that three participants could not justify their answer or did not fully recognise the cause of problem at the end of the session. This condition indicates that these participants tried to solve the problem without generating insight but by using intuition. This finding will be taken into account when analysing the solution rate, which indicates the insight occurrence, in the next section. The only participant who had inaccurate problem understanding was in the DES group. On the other hand, the two participants that had incomplete problem understanding were in. the ABM and DES groups. Most of participants had complete problem understanding and were able to provide solutions by understanding the existing problems. These participants were considered to have solved the problem by generating insight.

\subsection{Discontinuity in Thinking}

Discontinuity in thinking was observed based on the number of scenarios built by the participants. According to Gogi et al. (2016), if the participants can solve the problem correctly on the first attempt, they have not shown discontinuity in thinking. This implies that the participant tried to solve the problem without generating insight because they knew how to solve the problem, not through the use of simulation. In this study, the number of scenarios that were built by the participants can be seen in Table 3.

Table 3 Number of Scenarios Built

\begin{tabular}{l||cccc}
\hline $\begin{array}{c}\text { Number of } \\
\text { Scenario }\end{array}$ & Median & $\begin{array}{c}\text { DES } \\
\text { (lower-upper quartiles) }\end{array}$ & Median & $\begin{array}{c}\text { ABM } \\
\text { (lower-upper quartiles) }\end{array}$ \\
\hline User & 3 & $(1-5)$ & 5 & $(1-13)$ \\
Non-user & 2 & $(1-4)$ & 6 & $(1-13)$ \\
\hline
\end{tabular}

The experiment results showed that among both users and non-users in DES and ABM, there were participants that only built one scenario and submitted that scenario as the optimal solution. This indicates that there were participants who solved the problem on their first attempt without requiring the help of the simulation model. These participants were considered not to have discontinuity in thinking because they knew how to solve the problem after reading the case study. This finding will be taken into account when analysing the solution rate, which indicates insight occurrence, in the next section. 
In addition, statistical analysis using the chi-square test was also conducted in order to determine the difference in the number of scenarios built by users and non-users of DES and ABM. The results showed a p-value of 0.5896 . By using the significance level of 0.05 , it can be concluded that there was no significant difference between the number of scenarios built by users and non-users of DES and ABM.

\subsection{Change in Problem Understanding}

Change in participants' problem understanding was measured based on a self-assessment completed by participants. In the post-test questionnaire, the participants were asked about their change in understanding after attempting to solve the problem using the simulation model. The question used a five-point Likert scale with 1 indicating "A lot worse" and 5 indicating "A lot better". The results for participants" change in problem understanding are presented in Table 4.

Table 4 Participants' Self-assessment of Change in Understanding

\begin{tabular}{l||cccc||c}
\hline \multirow{2}{*}{ Self-Assessment } & \multicolumn{2}{c|}{ DES $(\mathbf{n}=\mathbf{1 7})$} & \multicolumn{2}{c||}{ ABM $(\mathbf{n}=\mathbf{2 0})$} & \multirow{2}{*}{ Total } \\
& Users & Non-users & Users & Non-users & \\
\hline A lot better & 3 & 2 & 1 & 1 & 7 \\
Better & 5 & 4 & 8 & 9 & 26 \\
Similar & 1 & 2 & 1 & 0 & 4 \\
Worse & 0 & 0 & 0 & 0 & 0 \\
A lot worse & 0 & 0 & 0 & 0 & 0 \\
\hline \hline Total & 9 & 8 & 10 & 10 & 37 \\
\hline
\end{tabular}

Most of the participants reported that they had better problem understanding after attempting to solve the problem using the simulation model. However, from Table 4 it can be seen that four participants claimed that their understanding remained the same before and after attempting to solve the problem using simulation. This could be because participants believed that they knew how to solve the problem after reading the case study. It could also be because the participants tried to solve the problem using intuition. The participants who claimed to have similar problem understanding were considered to have attempted to solve the problem without generating insight. This finding will be taken into account when analysing the solution rate in the next section.

After analysing the four variables, the rates of insight occurrence were calculated. This was done by combining the insight occurrence results for the four variables as presented in the previous section. The result of task performance analysis showed that there are $90 \%$ DES users, $73 \%$ DES non-users, all $\mathrm{ABM}$ users, and all $\mathrm{ABM}$ non-users who can solve the problem by generating insight. Furthermore, the results of the change in problem understanding show that $11 \%$ DES users, $12.5 \%$ DES non-users, and $10 \%$ ABM non-users attempt to solve the problem without generating insight.

After analysing the discontinuity in thinking of participants, it could be concluded that there were some participants who solved the problem without generating insight. Based on the participants' change in problem understanding, it was established that $11 \%$ DES users, $25 \%$ DES non-users, and $10 \% \mathrm{ABM}$ users solved the problem without generating insight. To summarise these results, the solution rates indicating insight occurrence for users and non-users of DES and ABM are presented in Table 5.

Table 5 Solution Rates Indicating Insight Occurrence

\begin{tabular}{cccc}
\hline $\begin{array}{c}\text { Solution rates, indicating } \\
\text { insight occurrence }\end{array}$ & $\begin{array}{c}\text { DES } \\
(\mathbf{n}=\mathbf{1 7})\end{array}$ & $\begin{array}{c}\text { ABM } \\
(\mathbf{n}=\mathbf{2 0})\end{array}$ & $\begin{array}{c}\text { Significance of } \\
\text { Difference }\end{array}$ \\
\hline Users & $50 \%(5 / 10)$ & $60 \%(6 / 10)$ & No \\
Non-users & $55 \%(6 / 11)$ & $60 \%(6 / 10)$ & $(\mathrm{p}$-value $=0.7215)$ \\
\hline
\end{tabular}


The results above show that $50 \%$ of DES users and $60 \%$ of ABM users solved the problem by generating insight. Even though the non-users of DES and ABM had not studied the simulation techniques, $55 \%$ of DES non-users and $60 \%$ of ABM non-users could solve the problem by generating insight. However, users and non-users of $\mathrm{ABM}$ had higher solution rates, indicating greater occurrence of insight than users and non-users of DES. This could be because ABM has a simpler interface; the scenario changes can also be made easily, simply by changing the parameters that are shown in the model interface.

A statistical test was conducted in order to calculate the differences between users and non-users of DES and ABM. Using the Fisher exact test, a p-value of 0.7215 was determined. With a significance level of 0.05 , it was concluded that there was no significant difference between users and non-users of $\mathrm{DES}$ and $\mathrm{ABM}$ in solving the given problem by generating insight.

\section{CONCLUSION}

From the discussion in previous sections, it can be concluded that, statistically, there is no significant difference in insight-generation by users and non-users of DES and ABM. However, users and nonusers of ABM showed higher solution rates, indicating the occurrence of insight, than users and nonusers of DES. However, this study applied reused models, meaning the participants were not required to develop their own model but to solve the problem using the given model. Additionally, the systems simulated both in DES and ABM in this study have low complexity only consisted of two to three parameters. Therefore, further research could analyse the difference in the model-building process between users and non-users of DES and ABM with the different complexity level.

\section{ACKNOWLEDGEMENTS}

The authors acknowledge the contribution of Hersetiawan et al. (2019) in regard to the student corner model in Flexsim software, and Wilensky (1997) in regard to the HIV model in the Netlogo library. Sections of this paper are based on Gogi, Tako and Robinson's (2016) experimental investigation into the role of simulation models in generating insights, published in European Journal of Operational Research, (pp. 931-944).

\section{REFERENCES}

Aalst, W. M. Van Der \& Voorhoeve, M. (2000). Simulation Handbook. Eidhoven: Technical University Eindhoven, Eindhoven.

Babulak, E., \& Wang, M. (2010). Discrete Event Simulation: State of the Art, Discrete Event Simulations. Aitor Goti (Ed.), InTech.

Bennett, N. D., Croke, B. F., Guariso, G., Guillaume, J. H., Hamilton, S. H., Jakeman, A. J., \& Pierce, S. A. (2013). Characterising performance of environmental models. Environmental Modelling \& Software, 40, 1-20.

Chung, C. A. (2004). Simulation Modeling Handbook: A Practical Approach. CRC Press.

Dubiel, B., \& Tsimhoni, O. (2005). Integrating agent-based modeling into a discrete event simulation. Proceedings of the 2005 Winter Simulation Conference, 1029-1037

Gogi, A. (2016). Insight Generation in Simulation Studies: An Empirical Exploration. Loughborough University.

Gogi, A., Tako, A. A., \& Robinson, S. (2016). An experimental investigation into the role of simulation models in generating insights. European Journal of Operational Research, 249(3), 931-944.

Hersetiawan, A., Sunggoro, A. M., Alsana, S., Puspita, S., Pramudita, S. I., \& Gani, Y. A. B. (2019). Simulasi Student Corner UGM menggunakan Software Flexsim.

Leemis, L., \& Park, S. (2004). Discrete-Event Simulation: A First Course. Prentice Hall. 4-17.

Macal, C. M., \& North, M. J. (2010). Tutorial on agent-based modelling and simulation. Journal of Simulation, 4(3), 151-162.

Majid, M. A., Fakhreldin, M., \& Zuhairi, K. Z. (2016). Comparing Discrete Event and Agent-Based Simulation in Modelling Human Behaviour at Airport Check-in Counters. International Conference on Human-Computer Interaction, 510-522. 
Pegden, C. D., Shannon, R. E., and R. P. Sadowski. 1990. Introduction to Simulation Using SIMAN. McGraw-Hill, Inc., New York.

Wilensky, U. (1997). Netlogo HIV model. http://ccl.nortwestern.edu/netlogo/models/HIV. Centre for Connected Learning and Computer-Based Modeling, Northwestern University, Evanston, IL.

Zankoul, E., Khoury, H., \& Awwad, R. (2015). Evaluation of Agent-Based and Discrete-Event Simulation for Modelling Construction Earthmoving Operations. 32nd International Symposium on Automation and Robotics in Construction and Mining,

\section{AUTHOR BIOGRAPHIES}

HILYA MUDRIKA ARINI holds a PhD in management science from the University of Strathclyde, Glasgow. She is an industrial engineering faculty member at Universitas Gadjah Mada, Indonesia. Her research interests focus on behavioural operations research and decision analysis.

NUR AINI MASRUROH is an associate professor in the Industrial Engineering Programme, Universitas Gadjah Mada, Indonesia. Her primary research interest is in operations research, decision making under uncertainties, healthcare system simulation and optimisation, production planning, inventory and scheduling, and revenue management.

NURUL LATHIFAH received her bachelor's degree in industrial engineering from Universitas Islam Indonesia in 2017 and an M.Sc degree in industrial engineering from Universitas Gadjah Mada in 2020. Her master's degree research focused on the implementation of behavioural operational research concepts in simulation. Currently, she is a research assistant focusing on research on routing problems at Universitas Gadjah Mada. Her main research interests include behavioural operational research, simulation, supply chain, and vehicle routing problems.

LAELA DEWI MAHARANI has completed her studies in industrial engineering at Universitas Gadjah Mada. She was an assistant lecturer in optimisation mathematics and is interested in conducting behavioural operations research. 